\title{
Effect of cranioplasty on functional and neuro- psychological recovery after severe acquired brain injury: fact or fake? Considerations on a single case
}

\author{
Francesco Corallo, PsyD \\ Angela Marra, MD \\ Placido Bramanti, MD \\ Rocco Salvatore Calabrò, MD, PhD
}

IRCCS Centro Neurolesi "Bonino-Pulejo", Messina, Italy

Correspondence to: Rocco Salvatore Calabrò

E-mail: salbro77@tiscali.it

\section{Summary}

Cranioplasty is performed, using autograft materials, to treat the "trephined syndrome" in patients previously submitted to craniectomy. Indeed, considerable improvements in neuropsychological deficits, control of convulsions and partial prevention of cerebral atrophy are achieved after this surgical procedure. We describe the pre- and post-operative neuropsychological and functional evaluation of a 30 -year-old male patient who underwent cranioplasty following previous craniectomy for a subarachnoid hemorrhage due to a spontaneously ruptured aneurysm of the right middle cerebral artery.

KEY WORDS: cognitive rehabilitation, cranioplasty, neuropsychological evaluation, severe brain injury

\section{Introduction}

Decompressive craniectomy is the standard surgical treatment for malignant cerebral edema and brain herniation resulting from cerebral infarction, intracranial hemorrhage and severe traumatic brain injury (Jung et al., 2012). After decompressive craniectomy, cranioplasty is also generally performed either for cosmetic reasons or to afford protection against the development of the syndrome of the trephined, i.e. neurological deterioration following the decompressive procedure. Despite being a relatively straightforward procedure, cranioplasty seems to be remarkably prone to complications (Schuss et al., 2012). Indeed, infection of the cranioplasty site has been shown to increase craniectomy-related morbidity and can lead to long-term use of antibiotics or removal of the graft material and subsequent repeat cranioplasty (Liang et al., 2007). After decompressive craniectomy, cranial defects are repaired with cryopreserved or artificial bone grafts at different time intervals. A neuropsychological assessment is the best approach for understanding the nature, the severity, and the modality of cognitive complaints. When cognitive complaints are reported or persist following brain injury, neuropsychological testing is useful for addressing diagnostic issues as well as treatment and rehabilitation planning. The process typically begins with a clinical interview, and then continues with tests that assess function in various cognitive and emotional domains. Because the effects of brain injury can have wide-ranging neurological, psychological and psychosocial consequences, both patients and caregivers can benefit from an assessment that identifies and quantifies deficits. Therefore, a neuropsychological assessment is an important measure of outcome and much more representative of the prognosis of neurosurgical patients than other outcome scales (Mokri, 2010). In the present study, we address the neuropsychological assessment of a patient undergoing cranioplasty for a large cranial defect.

\section{Case report}

An otherwise healthy 30-year-old male patient was admitted to a neurosurgery unit after a subarachnoid hemorrhage due to a spontaneously ruptured aneurysm of the right middle cerebral artery. A right frontotemporoparietal craniectomy with evacuation of the hematoma and coiling of the aneurysm was performed as initial treatment and the bone flap was not initially replaced. Consequently, the skin of the right temporoparietal region was markedly sunken due to the large skull defect. After around 20 days, the patient was admitted to our neurorehabilitation institute to undergo intensive motor and neurobehavioral training. When observed by us, the patient presented with a severe left hemiparesis with dysesthesia; moreover, he showed a severely depressed mood with anhedonia, irritability and sleep alterations. The high risk of recurrent trauma as well as the impaired cosmetic appearance and rapid cognitive decline observed in this patient indicated an urgent need for cranioplasty, which was performed about 30 days after the patient's admis- 
sion to our center. Indeed, after replacement of the bone flap the cognitive and neurological deficits improved within 40 days. The neuropsychological evaluation was performed a week before cranioplasty (baseline T0), one week after cranioplasty (T1), and around three months (T2) after cranioplasty, and it consisted of a psychosocial structured interview, the MiniMental Status Examination (MMSE), a depression and anxiety scale questionnaire (Hamilton Rating Scale for Depression and Anxiety), activities of daily living and instrumental activities of daily living scales (ADL and IADL), category verbal fluency and letter verbal fluency tasks, the Trail Making Test, Rey Auditory Verbal Learning Test immediate and delayed recall, and tests evaluting ideomotor apraxia and constructional apraxia. The neuropsychological evaluation performed before the cranioplasty (TO) showed severe cognitive deterioration, which partly improved immediately after cranioplasty (T1). However, a good functional recovery was seen at discharge (T2) (Table I). Interestingly, the improvements were more significant in memory function, information processing speed and inhibitory control, language function and visual-constructive abilities; some quantitative improvements were also seen in executive functions. Moreover, at discharge the patient was able to walk with monolateral support and his hemiparesis as well as his mood had improved.

\section{Discussion}

This case provides clear evidence of the possibility of recovery of cognitive, behavioral and motor function after a severe acquired brain injury, this recovery being partially related to an adequate cranioplasty. During the initial stages of a brain injury, there occur variable degrees of irreversible damage to the central nervous system, commonly known as the primary injury. Subsequently, a chain of events is set in motion leading to ongoing injury to the brain caused by edema, hypoxia and ischemia, which occur as a result of increased intracranial pressure (ICP), the release of toxic amounts of excitatory neurotransmitters such as glutamate, and impaired ion homeostasis (Lavinio et al., 2007). Acute brain injury treatment therefore focuses on preventing or minimizing the extent of secondary injury by targeting intracranial hypertension, oxygenation, and ion homeostasis in order to reduce cellular injury. Removal of skull sections has been suggested as a drastic measure for the management of elevated ICP unresponsive to other therapies. It is thought that surgical decompression could improve the damage (delayed brain damage) due to secondary causes, such as high ICP and reduced oxygenation of the brain. In a recent meta-analysis, the authors identified two types of surgical decompression: prophylactic or primary decompression and therapeutic or secondary decompressive craniectomy; the former is performed as a preventive measure against expected increases in ICP, while the latter is performed to control high ICP "refractory to maximal medical therapy" (Sahuquillo and Arikan, 2006). However, the debate over whether and when to perform these surgical procedures continues. Of course, any surgical procedure is associated with inherent risks. The majority of decompressive techniques are performed for the evacuation of a mass lesion (Compagnone et al., 2005). Once decompression has been decided upon, resection of a bone fragment large enough to allow dural expansion with less risk of herniation is generally recommended. Therapeutic decompressive craniectomy is only performed after other therapeutic measures (CSF drainage, moderate hypocapnia, mannitol, barbiturates, hyperventilation, hypothermia,

Table I - Neuropsychological assessment performed before (T0) and after (T1 and T2) cranioplasty.

\begin{tabular}{|c|c|c|c|c|c|c|c|c|c|}
\hline & \multicolumn{3}{|c|}{ T0 } & \multicolumn{3}{|c|}{$\mathrm{T} 1$} & \multicolumn{3}{|c|}{ T2 } \\
\hline & PS & CS & ES & PS & CS & ES & PS & CS & ES \\
\hline MMSE & 11 & 10.4 & 0 & 18 & 18.9 & 2 & 25 & 25.9 & 3 \\
\hline HRSA & 19 & & 0 & 14 & & 2 & 10 & & 3 \\
\hline HRSD & 18 & & 0 & 16 & & 1 & 12 & & 3 \\
\hline RAVLT-immediate recall & 17 & 23.10 & 0 & 26 & 26.8 & 3 & 38 & 36.10 & 3 \\
\hline RAVLT-delayed recall & 0 & 0 & 0 & 5 & 5.6 & 2 & 9 & 7.16 & 3 \\
\hline MOTOR LEARNING REVERSED & 12 & & 0 & 16 & & 2 & 24 & & 4 \\
\hline LVF & 14 & 22 & 0 & 16 & 13 & 1 & 25 & 22 & 2 \\
\hline IA (right) & 8 & & 0 & 10 & & 1 & 20 & & 4 \\
\hline $\mathrm{CA}$ & 11 & 9.75 & 1 & 20 & & 2 & 24 & & 4 \\
\hline TMT-A & 230 & 227 & 0 & 200 & 190 & 0 & 110 & 95 & 1 \\
\hline TMT-B & 0 & 0 & 0 & 500 & 458 & 0 & 300 & 287 & 0 \\
\hline TMT-B-A & 0 & 0 & 0 & 300 & 250 & 0 & 190 & 190 & 0 \\
\hline ADL & $1 / 6$ & & 0 & $3 / 6$ & & 4 & $6 / 6$ & & 4 \\
\hline IADL & $2 / 5$ & & 0 & $3 / 5$ & & 4 & $5 / 5$ & & 4 \\
\hline
\end{tabular}

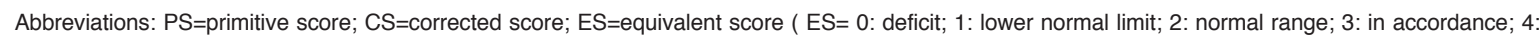
fully in accordance); MMSE=Mini-Mental State Examination; HRSA=Hamilton Rating Scale for Anxiety; HRSD=Hamilton Rating Scale for Depression; RAVLT=Rey Auditory Verbal Learning Test; LVF=letter verbal fluency; IA=ideomotor apraxia; $C A=$ contructional apraxia; TMT-A =Trail Making Test Part A; TMT-B =Trail Making Test Part B; TMT-B-A =Trail Making Test Part B minus Part A; ADL=activities of daily living; IADL=instrumental activities of daily living. 
etc.) have failed to control ICP (Morgalla et al., 2008). The potential improvements in neuropsychological function after cranioplasty have been shown in several studies. Some authors suggested that, after decompressive surgery, atmospheric pressure is transmitted to the cranial cavity, causing inward rotation of the scalp at the cranial defect (Sarov et al., 2010). This pressure acting on the cerebral cortex may cause neurological deficits. Maekawa et al., using ${ }^{133} \mathrm{Xe}$ CT scanning, reported that cranioplasty after decompressive craniectomy might increase cerebral blood flow (CBF) not only in the symptomatic hemisphere, but also in the other hemisphere. Although transcranial Doppler does not directly measure CBF, it does measure blood flow velocity in the basal intracranial arteries, through a different window (Maekawa et al., 1999). Our results confirmed previous reports that cranioplasty significantly improved neuropsychological status, with regard to memory function, processing speed and inhibitory control, language function and visual-constructive ability. For example, on the MMSE, which is a comprehensive assessment of cognitive function, the patient achieved a score of $11 / 30$ at T0 and $25 / 30$ at T2, an improvement of 14 points. Moreover, we also found that the ADL (T0 1/6, T2 6/6) and IADL (T0 2/5, T2 5/5) scores, which are functional scores of daily activities, improved significantly. Cranioplasty improved CBF velocities in all major intracranial arteries, not only on the side of the lesion adjacent to the cranioplasty, but also in distant regions, such as the contralateral hemisphere (Khrisnan et al., 2006). Thus, the neurological improvement after cranioplasty may be due to the increase in CBF velocities in all vessels, on both the lesional and the non-lesional side, resulting from elimination of the effects of atmospheric pressure on the brain (Schirmer et al., 2008).

In the patient here described, intensive and multidisciplinary rehabilitation programs were initiated early and then continued uninterruptedly, even throughout the patient's limited period of impaired consciousness. Nevertheless, we may argue that part of the cognitive and motor recovery, occurring within a week of cranioplasty, was related to this surgical procedure. Thereafter, since the improvement was gradual, the good cognitive and functional outcome was probably due to a competitive effect between cranioplasty and rehabilitation.

Indeed, other than these two speculative causes (cranioplasty and rehabilitation), an atypical time course of spontaneous recovery (Tsaousides and Gordon, 2009) is the only explanation that can be considered for the described late recovery of consciousness. The repair of cranial defects seems to offer patients clear benefits in terms of neurological improvement in many cognitive domains as well as in quality of life (Agner et al., 2002; Di Stefano et al.,
2012; Stiver et al., 2008). Nevertheless, larger sample studies should be encouraged in order to confirm these promising findings.

\section{References}

Agner C, Dujovny M, Gaviria M (2002). Neurocognitive assessment before and after cranioplasty. Acta Neurochir (Wien) 144:1033-1040.

Compagnone C, Murray GD, Teasdale GM, et al (2005). The management of patients with intradural post-traumatic mass lesions: a multicenter survey of current approaches to surgical management in 729 patients coordinated by the European Brain Injury Consortium. Neurosurgery 57: 1183-1192.

Di Stefano C, Sturiale C, Trentini P, et al (2012). Unexpected neuropsychological improvement after cranioplasty: a case series study. Br J Neurosurg 26:827-831.

Jung HJ, Kim DM, Kim SW (2012). Paradoxical transtentorial herniation caused by lumbar puncture after decompressive craniectomy. J Korean Neurosurg Soc 51: 102-104.

Khrisnan P, Bhattacharyya AK, Sil K, et al (2006). Bone flap preservation after decompressive craniectomy - experience with 55 cases. Neurol India 54:291-292.

Lavinio A, Timofeev I, Nortje J, et al (2007). Cerebrovascular reactivity during hypothermia and rewarming. $\mathrm{Br} \mathrm{J}$ Anaesth 99:237-244.

Liang W, Xiaofeng Y, Weiguo L, et al (2007). Cranioplasty of large cranial defect at an early stage after decompressive craniectomy performed for severe head trauma. J Craniofac Surg 18:526-532.

Maekawa M, Awaya S, Teramoto A (1999). [Cerebral blood flow (CBF) before and after cranioplasty performed during the chronic stage after decompressive craniectomy evaluated by Xenon-enhanced computerized tomography (Xe-CT) CBF scanning]. No Shinkei Geka 27:717-722.

Mokri B (2010). Orthostatic headaches in the syndrome of the trephined: resolution following cranioplasty. Headache 50 : 1206-1211.

Morgalla $\mathrm{MH}$, Will BE, Roser F, et al (2008). Do long-term results justify decompressive craniectomy after severe traumatic brain injury? J Neurosurg 109:685-690.

Sahuquillo J, Arikan F (2006). Decompressive craniectomy for the treatment of refractory high intracranial pressure in traumatic brain injury. Cochrane Database Syst Rev (1) CD003983.

Sarov M, Guichard JP, Chibarro S, et al (2010). Sinking skin flap syndrome and paradoxical herniation after hemicraniectomy for malignant hemispheric infarction. Stroke 41:560562.

Schirmer CM, Ackil AA Jr, Malek AM (2008). Decompressive craniectomy. Neurocrit Care 8:456-470.

Schuss P, Vatter H, Marquardt G, et al (2012) Cranioplasty after decompressive craniectomy: the effect of timing on postoperative complications. J Neurotrauma 29:1090-1095.

Stiver SI, Wintermark M, Manley GT (2008). Reversible monoparesis following decompressive hemicraniectomy for traumatic brain injury. J Neurosurg 109:245-254.

Tsaousides T, Gordon WA (2009). Cognitive rehabilitation following traumatic brain injury: assessment to treatment. Mt Sinai J Med 76:173-181. 